\title{
The effect of Social licence on Dynamic Decisions making: a Case Study of a Gold Mine
}

\author{
$\underline{\text { W. Chen }}^{\mathrm{a}}$, N. Langrené ${ }^{\mathrm{a}}, \mathbf{Z}$. Zhu ${ }^{\mathrm{a}}$

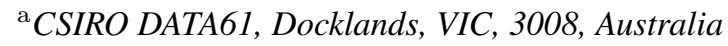 \\ Email: Wen.Chen@csiro.au
}

\begin{abstract}
Dynamic decision making can significantly add value to mining projects and deliver more consistent outcomes under conditions of uncertainty. To achieve consistent and better outcomes, decision-makers in industry can benefit from an analytical optimal decision support tool that will help them to formulate and reach the optimal outcomes. The dynamic decision support tool developed at CSIRO is based on novel stochastic optimal control methodologies that can optimise decisions in situations with multiple uncertain variables and decisions. The types of market uncertainties that can be considered include commodity prices, interest rates and exchange rate, while uncertainties considered for mineral ore bodies include reserve levels, boundaries between ore types and even geo-metallurgical parameters. Social license to operate (SLO), the acceptance and approval level, is crutial for mining companies during the mining development. This paper focuses on the impact of social licence in making optimal dynamic decisions, as illustrated by a case study of a gold mine.

The study examines the optimal extraction of gold using the mining characteristics, operating costs and salaries from a stylised gold mine in West Africa. We assume that the company has the operational flexibility to temporarily stop, restart or completely abandon extraction in response to gold price movement. Empirical evidence suggests that closing the mine may meet a very negative response from the local community. This in turn may lead to considerable future difficulties with hiring workers when reopening the mine, which can be viewed as the loss of the social licence to operate. To avoid such difficulties, management may consider paying salaries (full or partial) to local workers during a mine closure. While such a strategy may be expensive, it could help to maintain the community's trust and thus retain the social licence to operate. Consequently, such an expensive strategy will significantly reduce the switching costs when re-opening the mine and the total value of the mine can actually be increased through such an strategy.
\end{abstract}

This paper is the first attempt (to our best knowledge) to introduce social licence into the optimal flexible management of a mine extraction project. It introduces a simple conceptual model of the social response to managerial actions in the form of switching costs. In addition to maintenance costs, the company is assumed to pay local workers a proportion of their salary in order to prevent negative social response and to maintain its SLO. In the worst case, if no salary is paid to the local workers, the social licence would be lost, preventing the mine from being reopened. If the mine is closed permanently, the company has to pay decommissioning costs. It is also assumed that the company makes a one-off payment to national and international workers when they are laid off. The paper also illustrates how the dynamic decision support tool can assist industry to determine the optimal strategy and determine the optimal proportion of the salary to pay local workers during a mine closure.

Keywords: Real options, social licence to operate, stochastic optimal control, switching boundaries 


\section{INTRODUCTION}

Dynamic decision making can add significant value to natural resource extraction projects and deliver better and more consistent outcomes when a large degree of uncertainty exists. Typical uncertainties or risks are: financial risks (eg. commodity prices, interest rates and exchange rate); geological risks (eg. reserves, ore grade); and social risks which can be measured as the value of social licence to operate (henceforth SLO). The effects of financial and geological uncertainties have been extensively studied in the literature, particularly in the mathematical finance area. It is now well known in the minerals industry that the flexibility to revise managerial and operational decisions over time in response to uncertain market conditions can significantly increase the value of a project. Since the seminal paper of Brennan and Schwartz [1985] (henceforth B\&S), the consensus is that the value of flexibility to delay, temporarily close, reopen and completely abandon a mineral extraction project in response to commodity price fluctuations can be significant. Such flexibility offers ample opportunity for optimal management strategies in natural resource investments, and not surprisingly, also creates research interest using either deterministic methods from operational research or stochastic methods from stochastic control or real option (see, e.g., Trigeorgis [1996], Slade [2001], Dimitrakopoulos and Abdel Sabour [2007], Carmona and Ludkovski [2008], Tsekrekos et al. [2012], Bao et al. [2013], Mortazavi-Naeini et al. [2014], Tarnopolskaya et al. [2015], Chen et al. [2015], Chen et al. [2016]).

Social and political risks are another type of uncertainty that can significantly impact on natural resource extraction projects. In particular, the profitability of mining companies is directly affected by SLO, which is defined as the acceptance and approval of a mining development by the local community and other stakeholders. The risk for social acceptance or the lack of it can be represented by the acceptance level of SLO. When a mining operation develops its legitimacy and credibility with local stakeholders, the acceptance and approval level (SLO) will increase. If full trust between the local community and the mining company is established, the SLO can reach the level of psychological identification for the two sides (Boutilier and Thomson [2011]), for which the social-political risk becomes extremely low for the projects. On the other hand, when the SLO is limited, lost or withdrawn, the extraction will be regarded as illegitimate by the local community, and thus will have to be temporarily or even permanently suspended. Losing a SLO creates substantial social-political risk. Boutilier and Thomson [2011] propose a cumulative hierarchical model to describe SLO as a continuous process of social capital development. While most of the early theoretical studies on this topic are descriptive and qualitative, a few quantitative studies have recently been performed. In Moffat and Zhang [2014], the authors measure and model the critical elements of a SLO via social psychological research in intergroup relations. They propose using community trust as the central element of the SLO modelling, and use a 5-point descriptive statistics scale and bivariate correlations to analyse how the key variables relate to each other and how a mining company can manage and mitigate operational impacts. In Zhang and Moffat [2015], the authors examine the local community's perception of the balance between the beneficial and negative impacts of mining, and how this affects the SLO of a mining company. In Tarnopolskaya and Littleboy [2015], the authors discuss the possibility of the quantitative modelling of SLO and propose several quantitative methods including the conceptual framework for dynamic financial valuation of SLO.

So far there is no literature on mining project valuation that takes financial and geological uncertainties together with social-political risk into account in a quantitative manner. Doing so requires treating SLO as a sort of option on social risk, i.e. an investment aimed at reducing social risk, in particular the worst outcomes such as forced closure. Such an approach of linking SLO with Real Option has been suggested in Nelsen [2007], and later conceptualised in Tarnopolskaya and Littleboy [2015]. The present conference paper follows the conceptual framework for dynamic financial valuation of SLO of Tarnopolskaya and Littleboy [2015], and incorporates some aspects of SLO into a quantitative and stochastic approach for decision making under uncertainty in the lines of Brennan and Schwartz [1985] as applied to the valuation of a mine extraction project. In general, the value of a mining project depends on the uncertain future metal price, remaining reserve and how the company mitigates social risk and maintains SLO during operation. As a simple model for the development and maintenance of SLO for a dynamically managed mine, we focus on employment opportunity for local workers and on life-of-mine rehabilitation. The classical real option literature emphasizes the value provided by the possibility of temporary mine closure when prices are low, but neglects the effect of such closures for local workers and SLO. In order to keep such flexibility while maintaining SLO, we assume that the mining company has the option to smooth the effect of a downturn on local workers by paying a partial salary to them during temporary shutdowns. Such a decision can maintain trust and reputation with local staff and community, so as to ensure human resource availability when reopening the mine. For managers, it is critical to have an estimation of the potential losses and gains when deciding on the proportion of salary to 
pay. The second SLO feature included in the model is rehabilitation costs at the end of the life of the mine, which are critical for the reputation of the company and its social licence to operate other mines.

We model the mine project valuation as a stochastic control problem and solve it numerically using the Regression Monte Carlo method (Chen et al. [2015], Langrené et al. [2015], Chen et al. [2016]). The solution outputs are: 1) the optimal expected mine value, and 2) the actual operational policy over the life of the mine, for each fixed proportion of salary paid during the mine closure. The optimal operational policy can assist a mine manager to identify the best decision to make at each future decision time based on the information available at that time, e.g. for any particular future metal price and ore remaining reserve. It can also help the manager to establish the optimal salary proportion to be paid during temporary closures. This methodology presented here is a novel way of combining multiple risk variables with the value of SLO. To illustrate the potential benefit, we implement the methodology for a stylised gold mine in West Africa. For easy analysis of the computed outputs, we display in an intuitive way the information about the boundaries between the different decision regimes which evolve over time. Such boundaries, also known as switching boundaries, or critical (threshold) curves, have been studied in a number of applications in both deterministic and stochastic optimal control problems (see, e.g., Carmona and Ludkovski [2008], Tarnopolskaya and Fulton [2010b], Tarnopolskaya and Fulton [2010a], Mortazavi-Naeini et al. [2014], Tarnopolskaya et al. [2015]). The use of an intuitive display format for multivariate decision curves is important for the understanding and adoption of such complex dynamic real option tools by decision-makers in the industry.

The paper is organized as follows. The problem formulation is discussed in Section 2. The least squares Monte Carlo (LSMC) method and algorithm for construction of the switching surfaces are described in Section 3 . The numerical results, visualization of the switching surface and discussion are described in Section 4. Finally, the conclusions are given in Section 5.

\section{Problem Description ANd Formulation}

We study the optimal extraction on a West African gold mine from Ndiaye and Armstrong [2013], using the same mining characteristics, operating costs and salaries. The workforce consists of local, national and international workers (the breakdown and the salaries are set as in Ndiaye and Armstrong [2013]). There are three possible operating regimes of the mine: (1) open; (2) temporarily closed; and (3) abandoned. When the mine is open, the company has to pay the operation cost, which is proportional to productivity. When the mine is temporarily closed, the company has to pay maintenance costs. In addition, the company is assumed to pay local workers a proportion $p$ of their salary in order to prevent negative social response. Empirical evidence suggests that closing the mine may meet a very negative response from the local community, which in turn may lead to considerable difficulties with keeping the SLO and hiring workers when the mine needs to be reopened. To avoid such difficulties, managers may consider paying the salaries (full or partial) of local workers during the mine closure. While such a strategy may be expensive, it could help to maintain the community trust and the social licence, and will reduce the switching costs when reopening the closed mine. Therefore, we assume a simple functional form for the switching cost proportional to $1 / p^{3}$. This switching cost act as a penalty function to prevent from setting $p$ to zero. In the worst case, if no salary is paid to the local workers, the social licence would be lost, preventing the mine from being reopened in the future (this corresponds to infinite switching costs from closed to open in this model). If the mine is closed permanently (ie. abandoned), the company has to pay decommissioning fees. It is also assumed that the company makes a one-off payment to national and international workers when they are laid off.

We assume the company owns a mining licence for $T=15$ years. With the chosen extraction speed, the mine will be depleted in $T_{0}=10$ years if the company keeps the mine open continuously. When the licence expires or the mine is depleted, whichever comes first, this mining project is ended automatically. During the life of mine, the company has the operational flexibility in response to uncertain gold price $S$ and remaining reserve $Q$. Such a problem has been studied on an infinite time horizon by Brennan and Schwartz [1985] who formulated this problem as a quasi-variational inequality. Realistic mine planning problems usually have a finite time horizon $T$, as mining licences have finite lifetime. In this section, we formulate the problem as a discrete time, finite horizon stochastic switching problem. We assume that before depletion, the manager has the option to change operating regimes at pre-specified discrete decision times $t_{n}=n \Delta t, n=0,1, \ldots, N-1$, where $\Delta t=T / N$. We also assume that the ore grade is known for each mine level in $\Delta t$ using the data from Ndiaye and Armstrong [2013]. The grade of each level and the quantity of ore can be view as a series $\left\{\left(g_{l}, q_{l}\right)\right\}, l=1,2, \ldots, 10$ where $l$ is the inventory level. Denote as $G_{n}$ the inventory level at time $t_{n}$. Suppose that, at time $t_{n}, G_{n}=g_{l}$. Then, if at time $t_{n}$ the mine is open, $G_{n+1}=g_{l+1}$. If instead the mine is closed, 
the grade remains the same: $G_{n+1}=g_{l}$. Similarly, we define the quantity $Q_{n}$ at time $t_{n}$. The gold recovered at time $t_{n}$ is $q s_{n}:=Q_{n} \times G_{n}$. Define $\mathrm{R}$ as the remaining reserve process. $R_{0}$ is the total gold reserve. The discretised remaining reserve is given by $R_{n+1}=R_{n}-q s_{n} \Delta t \mathbb{1}\left\{i_{n}=o\right\}$. The gold price at $t_{n}$ is denoted $S_{n}$. In this case, we assume that the commodity price follows a one-dimensional geometric Brownian motion. In a discretised form, it is given by

$$
S_{n+1}=S_{n} e^{\left(r-\delta-\frac{\sigma^{2}}{2}\right) \Delta t+\sigma\left(W_{n+1}-W_{n}\right)}, \quad W_{n+1}-W_{n} \sim \mathcal{N}(0, \Delta t) \quad \text { i.i.d. }
$$

where $r$ is the risk-free rate, $\delta$ is the instantaneous convenience yield of the commodity, $\sigma$ is the volatility of the spot price. We can also implement mean-reversion process or other dynamics for the price.

We define the set of operating regimes as $\mathbb{Z}=\{o, c, a\}$, where $o, c$ and $a$ denote an open (operating), a temporarily closed and an abandoned mine respectively. The change of operating regime at time $t_{n}$ from the regime $i \in\{o, c\}$ to $j \in \mathbb{Z}$ incurs a switching cost:

- $k\left(t_{n}, o, c\right)=K_{0} e^{\pi t_{n}}$ (closing cost)

- $k\left(t_{n}, c, o, p\right)=K_{0} e^{\pi t_{n}} / p^{3}$ (reopening cost)

- $k\left(t_{n}, o, a\right)=k\left(t_{n}, c, a\right)=D_{0} e^{\pi t_{n}}$ (decommissioning and remediation costs)

where $\pi$ is the inflation rate. $k\left(t_{n}, i, i, p\right)=0$ if no switching occurs. The cash flow $\Pi_{i}\left(t_{n}, S_{n}\right)$ in the operating regime $i \in \mathbb{Z}$ between two decision times $\left[t_{n}, t_{n+1}\right), n=0, \ldots, N-1$ is given by

- For an open mine: $\Pi_{o}\left(t_{n}, S_{n}\right)=q s_{t_{n}}\left(S_{n}-A_{n}\right)$ - Tax $\left(S_{n}\right)$-Salary, where $A_{n}=A_{0} e^{\pi t_{n}}$ is the operating cost, Salary is the total salary, $\operatorname{Tax}\left(S_{n}\right)=p_{1} q s_{t_{n}} S_{t_{n}}+p_{2} q\left(S_{n}\left(1-p_{1}\right)-A_{n}\right)$ is the total income tax and royalties, with $p_{1}$ and $p_{2}$ denoting the royalty rate and the income tax respectively.

- For a closed mine: $\Pi_{c}\left(t_{n}, S_{n}\right)=-M_{n}$ - Salarylocal $\times p$, where Salarylocal is the salary for the unskilled local labor, $M_{0}$ is the initial maintenance cost, and $M_{n}=M_{0} e^{\pi t_{n}}$ is the after-tax maintenance cost.

- For an abandoned mine: $\Pi_{a}\left(t_{n}, S_{n}\right)=0$

We denote by $V\left(t_{n}, S_{n}, R_{n}, i, p\right)$ the value of the mine at $t_{n}$ when the commodity price is $S_{n}$, with $R_{n}$ reserves and status $i$ and the laid-off payment ratio is $p$, and assume that $V\left(T, S_{N}, R_{N}, i, p\right)=0$ (that is, the value is 0 after the expiration of mining licence). The goal of the mining company is to maximize the value $V\left(t_{0}, S_{0}, R_{0}, i, p\right)$ though an optimal strategy. The Bellman equation for this problem is given by

$$
V\left(t_{n}, S_{n}, R_{n}, i_{n^{-}}, p\right)=\max _{j \in \mathbb{Z}}\left\{\Pi_{j}\left(t_{n}, S_{n}\right) \Delta t-k\left(t_{n}, i_{n^{-}}, j, p\right)+\mathbb{E}\left[e^{-\tilde{r} \Delta t} V\left(t_{n+1}, S_{n+1}, R_{n+1}, j, p\right) \mid S_{n}, R_{n}\right]\right\}
$$

where $i_{n^{-}}$denotes the operating regime right before the decision time $t_{n}, \lambda$ is the property tax rate, proportional to the project value, and $\tilde{r}=r+\lambda$.

\section{NuMERICAL METHOD}

In this section, we describe how to use the Regression Monte Carlo method to maximise the value of this gold mine with an optimal policy for the whole life of the mine, with different proportion $p$ of salary paid to the local employee during temporary mine closures. From there the optimal proportion can be identified.

\subsection{Regression Monte Carlo Approach}

The Regression Monte Carlo approach is a versatile simulation-based technique, for which the continuation function in the Bellman equation (the last term in (2)) is approximated via basis functions. It was first introduced for American option pricing problems (Longstaff and Schwartz [2001]), and has become popular for stochastic control problems due to its flexibility and ease of implementation (see, e.g., Dimitrakopoulos and Abdel Sabour [2007], Tsekrekos et al. [2012], Bao et al. [2013], Tarnopolskaya et al. [2015], Chen et al. [2015], Chen et al. [2016]). The Regression Monte Carlo algorithm is composed of two parts:

1. A forward simulation loop: $M$ Monte Carlo paths of the commodity prices $\left\{S_{n}^{m}: m=1,2, \ldots, M ; n=\right.$ $0,1, \ldots, N\}$ are generated, with the same initial price $S_{0}^{m}=S_{0}$, using equation (1).

2. A backward induction loop: the continuation functions $\Phi\left(t_{n}, S_{n}, R_{n}, i\right)$ are computed by regressing the discounted realized cashflows along the simulated paths onto a set of basis functions $\left\{\phi_{l}\left(S_{n}^{m}\right)\right\}$ for each 
operating regime $i \in\{o, c\}$ and each feasible reserve level. At time $t_{n}$, for each simulated path $S_{n}^{m}$, a switch from the current operating regime $i$ to another operating regime $j$ happens if and only if

$$
\Pi_{i}\left(t_{n}, S_{n}^{m}\right) \Delta t+\Phi\left(t_{n}, S_{n}^{m}, R_{n}, i\right)<\max _{j \neq i}\left[\Pi_{j}\left(t_{n}, S_{n}^{m}\right) \Delta t-k\left(t_{n}, i, j, p\right)+\Phi\left(t_{n}, S_{n}^{m}, R_{n}, j\right)\right] .
$$

In this example, we use monomials up to order three as basis functions. Other types of regression such as local regressions could also be used. At the initial time $t_{0}=0$ no regression is needed (only simple expectations), and equation (3) provides the optimal initial decision.

\subsection{Constructing the Switching Surfaces}

The Bellman equation (2) reveals that the optimal operating regime depends on the previous operating regime, the current gold price, the remaining reserve, and the payment ratio $p$ during temporary mine closure. Following the switching set definition in Chen et al. [2015], we can construct the switching boundaries and switching surfaces for any given decision time during the entire mine life. The switching boundaries between the operating modes $i$ and $j$ are the critical prices $S_{i, j}^{*}\left(R_{n}\right)$ that trigger a regime switch from $i$ to $j$ when crossed. In our implementation, at each time step $t_{n}$, we perform one regression for every feasible reserve level and every operating mode using the whole set of Monte Carlo points ('hypothetical' states), rather than selecting the subset of paths corresponding to each reservel level and operating mode (as in Tarnopolskaya et al. [2015] and Chen et al. [2015]). By doing this, we increase the number of points in each switching set, and thus produce smoother switching boundaries. In Fig. 1, we can notice a hysteresis band which is due to switching costs. We repeat this computation for each $p$.

\section{Numerical Results and Discussion}

We use the data from Ndiaye and Armstrong [2013] and extend it to a 15-year mine project by interpolating the reserve and grade data. In this case study, the gold recovered above the cutoff in each open year is $\{q s\}=\{298.31,265.65,232.98,203.83,174.68,131.28,87.88,63.83,39.78,20\}$ koz. We equally divide the production by 2 to approximate a semi-annual production. The grade for each open period is $\{g\}=\{1.63,1.575,1.52,1.485,1.45,1.365,1.28,1.21,1.14,1.07\} \mathrm{g} / \mathrm{T}$. The operating cost (including extraction, treatment and administration cost) is $\$ 11.88 k$ per ton.

The salary for expatriates, highly skilled nationals and unskilled workers are $\$ 12000, \$ 2600$ and $\$ 1000$ per month respectively. In this case study, there are 18 expatriates, 130 skilled personnel and 367 unskilled workers. Royalties are $3 \%$ of revenue, the corporate tax rate is $30 \%$. The inflation rate is $8 \%$, the risk-free rate is $10 \%$ and the convenience yield is $1 \%$. We assume the gold price follows a geometric Brownian motion, with initial price $\$ 1000 / \mathrm{oz}$ and volatility $44.7 \%$.

Figure 1 illustrates the decision regions as functions of the remaining reserve and price for different proportions $p$ of salary paid, at the 10th year of the 15-year planning horizon. This figure shows four regions of optimal decisions: (1) open; (2) temporarily close; (3) abandon; (4) hysteresis region where no change of operating regime occurs. It also shows four switching boundaries (' $c \rightarrow o$, , $o \rightarrow c$, , $o \rightarrow a$, ' $c \rightarrow a$ '). The regions of optimal decisions (and therefore the optimal strategies) are strongly dependent on $p$.

1. When prices are high, the best decision is to keep the mine open (green). Conversely, when prices are very low, the best decision is to abandon the mine (blue). These observations are true for every p.

2. When prices are low but reserve is high, the best decision is to temporarily close the mine (grey). This closing period gives the mining company a chance to save the reserve in the hope of higher future gold price. As $p$ decreases, the 'closed' region shrinks and finally disappears completely at around $p=0.4$, meaning that closing the mine temporarily is not an option anymore due to the large social cost.

3. The switching boundary $c \rightarrow o$ (dark green) moves upwards as $p$ decreases. This is because the switching cost is inversely proportional to $p^{3}$ : the smaller $p$ the harder it is to reopen the mine.

4. The previous two observations can be summarized by the size of the hysteresis region (light green region between boundaries ' $o \rightarrow c$ ' and ' $c \rightarrow o$ '). The hysteresis region, in which no switching occurs, is due to switching costs (see discussions in Carmona and Ludkovski [2008], Tarnopolskaya et al. [2015], Chen et al. [2015]). This region grows as $p$ decreases, meaning that the lower the salary paid during temporary interruption period, the higher the switching cost and the harder to reopen the mine. Eventually, the option to close temporarily disappears altogether, reducing the management options to the timing of the end of life of the mine (optimal stopping problem). 

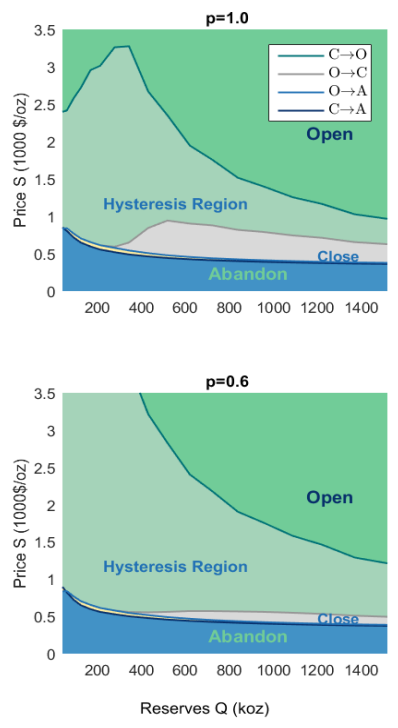

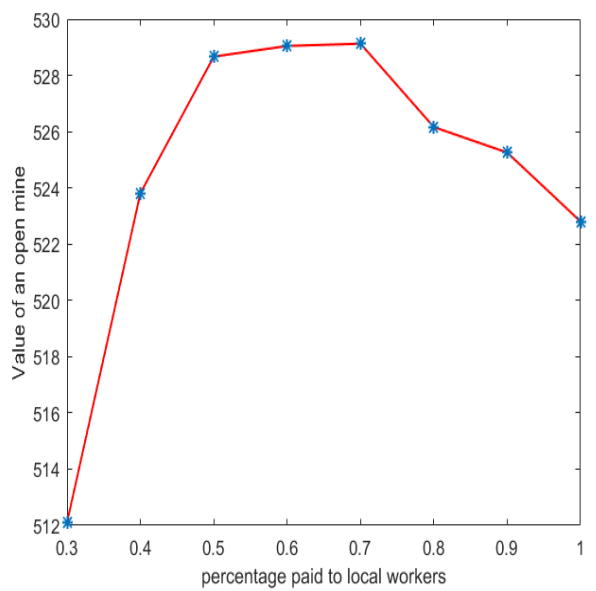

Figure 2. Mine value (\$millions) as a function of the proportion of salary paid $p$.

Figure 1. Decision Regions as functions of remaining reserve at 10th year for different proportion of the salary paid $p$.
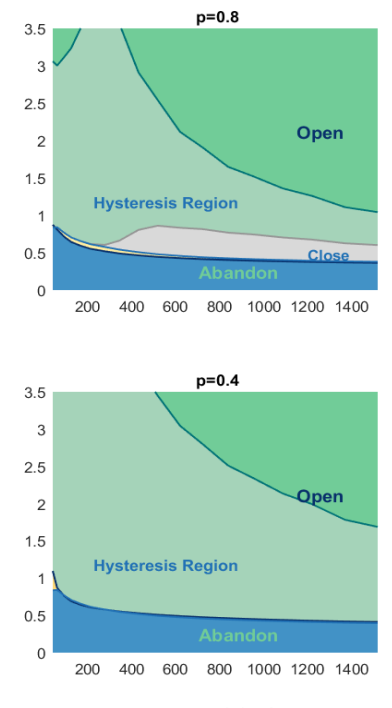

Reserve Q (koz)

Finally, we illustrate how the dynamic decision tool can be used to establish the optimal proportion of salary that the company should pay to local workers during mine closure. Figure 2 shows the value of the mine as a function of the proportion $p$ of salary paid. It suggests that the maximum value can be achieved between $p=0.5$ and 0.7 with our choice of parametric shape $k\left(t_{n}, c, o, p\right)$ for the reopening cost ' $c \rightarrow o$ '. However, the relative value difference with the safe $p=1.0$ case is less than $2 \%$, meaning that the company could decide to pay full salary even when the mine is closed to avoid potential social problems.

\section{Conclusion}

This paper revisits the real option approach for strategic mine planning, and focuses on the effect of social licence on the set of available management options. We consider the effect of exercising these options on the social licence to operate (SLO) of the mining company and propose a simple penalty model, in which the cost of reopening a mine depends on the proportion of salary paid to the local workers during the temporary shutdown (the lower the proportion, the harder to reopen). We use a test case based on a stylised gold mine from Ndiaye and Armstrong [2013]. The main result is that if SLO is ignored (little to no salary paid during closure), the option to temporarily close the mine completely disappears, reducing the management options and consequently reducing the mine value. This paper illustrates how SLO can affect management options, to the point where it can cancel some of them. Even though the chosen quantitative SLO model is simple and focuses on one single aspect of SLO, it opens the door to further quantitative analyses of SLO and its effect on strategic planning under uncertainty.

\section{ACKNOWLEDGEMENT}

We would like to thank Dr. Tanya Tarnopolskaya, former research scientist from Data61 CSIRO, for her supervision on this project.

\section{REFERENCES}

C. Bao, M. Mortazavi, S. Northey, T. Tarnopolskaya, A. Monch, and Z. Zhu. (2013). Valuing flexible operating strategies in nickel production under uncertainty. In MODSIM2013, 20th International Congress on Modelling and Simulations, pages 1426-1432, Australia, December 2013.

R. Boutilier and I. Thomson. (2011). Modeling and measuring the social license to operate: Fruits of a dialog between theory and practice. International Mine Management. 
W. Chen et al, Dynamic Decisions under Uncertainty: a Case Study of a Gold Mine

M. J. Brennan and E. S. Schwartz. (1985). Evaluating natural resource investment. The Journal of Business, 58(2):135-157.

R. Carmona and M. Ludkovski. (2008). Pricing asset scheduling flexibility using optimal switching. Applied Mathematical Finance, 15(5-6):405-447.

W. Chen, N. Langrené, and T. Tarnopolskaya. (2015). Switching surfaces for optimal natural resource extraction under uncertainty. In MODSIM2015, 21st International Congress on Modelling and Simulations, Australia, December 2015.

W. Chen, N. Langrené, and T. Tarnopolskaya. (2016). Natural resource extraction with production target: the real option value of variable extraction rate. In ROC2016, 20th Real Option Conference, 2016.

R. G. Dimitrakopoulos and S. A. Abdel Sabour. (2007). Evaluating mine plans under uncertainty: can the real options make a difference? Resources Policy, 32:116-125.

N. Langrené, T. Tarnopolskaya, W. Chen, Z. Zhu, and M. Cooksey. (2015) New regression Monte Carlo methods for high-dimensional real options problems in minerals industry. In MODSIM2015, 21st International Congress on Modelling and Simulations, Australia, December 2015.

F. Longstaff and E. Schwartz. (2001). Valuing American options by simulations: a simple least squares approach. Review of Financial Studies, 14(1):113-148.

Kieren Moffat and Airong Zhang. (2014). The paths to social licence to operate: An integrative model explaining community acceptance of mining. Resources Policy, 39:61-70.

M. Mortazavi-Naeini, T. Tarnopolskaya, and C. Bao (2014). Decision regions for natural resource investment under uncertainty. In Lecture Notes in Engineering and Computer Science: Proceedings of The World Congress on Engineering 2014, WCE 2014, pages 916-922, London, UK, July 2014.

A.A. Ndiaye and M. Armstrong. (2013). Evaluating a small deposit next to an economically viable gold mine in West Africa from the points of view of the mining company, the government and the local community. Resources Policy, 38(2):113-122.

J. Nelsen. (2007). Social license to operate: integration into mine planning and development. Master's thesis, University of British Columbia.

M. E. Slade. (2001). Valuing managerial flexibility: an application of real-option theory to mining investments. Journal of Environmental Economics and Management.

T. Tarnopolskaya and N. Fulton. (2010a). Synthesis of optimal control for cooperative collision avoidance for aircraft (ships) with unequal turn capabilities. Journal of Optimization Theory and Applications, 144(2):367-390.

T. Tarnopolskaya and N. Fulton. (2010b), Dispersal curves for optimal collision avoidance in a close proximity encounter. In Lecture Notes in Engineering and Computer Science: Proceedings of The World Congress on Engineering 2010, WCE 2010, pages 1789-1795, London, UK, July 2010.

T. Tarnopolskaya, W. Chen, and C. Bao. (2015). Switching boundaries for flexible management of natural resource investment under uncertainty. IAENG Transactions on Engineering Sciences, pages 1-14.

Tanya Tarnopolskaya and Anna Littleboy. (2015). Towards dynamic financial valuation of social licence to operate under uncertainty. 21st International Congress on Modelling and Simulation (MODSIM2015), 2015.

L. Trigeorgis. (1996). Real Options: Managerial Flexibility and Strategy in Resource Allocation. MIT Press, Cambridge.

A. E. Tsekrekos, M. B. Shackleton, and R. Wojakowski. (2012). Evaluating natural resource investment under different model dynamics: managerial insight. European Financial Management, 18(4):543-577.

Airong Zhang and Kieren Moffat. (2015). A balancing act: The role of benefits, impacts and confidence in governance in predicting acceptance of mining in australia. Resources Policy, 44:25-34, 2015. 